\title{
Barriers and Facilitators to Seek Help for Substance Use Disorder among Dutch Physicians: A Qualitative Study
}

\author{
Pauline M. Geuijen ${ }^{\mathrm{a}, \mathrm{b}}$ Esther Pars ${ }^{\mathrm{a}, \mathrm{b}}$ Joanneke M. Kuppens ${ }^{c}$ \\ Aart H. Schene ${ }^{a}$ Hein A. de Haan ${ }^{\text {b, d }}$ Cornelis A.J. de Jong ${ }^{\text {b, e }}$ Femke Atsma $^{f}$ \\ Arnt F.A. Schellekens ${ }^{a, b}$ \\ aDepartment of Psychiatry, Donders Institute for Brain, Cognition and Behaviour, Centre for Neuroscience, Radboud \\ University Medical Center, Nijmegen, The Netherlands; ${ }^{\text {b} N i j m e g e n ~ I n s t i t u t e ~ f o r ~ S c i e n t i s t-P r a c t i t i o n e r s ~ i n ~ A d d i c t i o n ~}$ \\ (NISPA), Nijmegen, The Netherlands; ${ }^{~ P h y s i c i a n ~ H e a l t h ~ P r o g r a m ~ A B S-D o c t o r s, ~ R o y a l ~ D u t c h ~ M e d i c a l ~ A s s o c i a t i o n ~}$

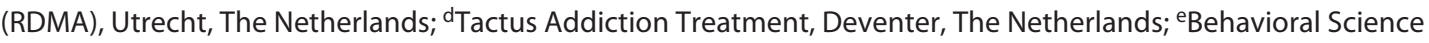 \\ Institute, Radboud University Nijmegen, Nijmegen, The Netherlands; ${ }^{f}$ Radboud Institute for Health Sciences, \\ Scientific Center for Quality of Healthcare (IQ Healthcare), Radboud University Medical Center, Nijmegen, The \\ Netherlands
}

\section{Keywords}

Barriers · Facilitators $\cdot$ Help seeking $\cdot$ Physicians $\cdot$ Substance use disorders

\begin{abstract}
Introduction: Substance use disorders (SUDs) among physicians affect their health, quality of life, but potentially also their quality of care. Despite the availability of effective specific Physician Health Programs (PHPs), physicians with SUD often experience barriers when seeking professional help. Therefore, we studied barriers and facilitators when seeking help for SUD among physicians from a multiple perspective approach. Methods: A qualitative design was adopted for 2 sub-studies. First, answers of 2 open-ended questions (about anticipated barriers and facilitators) of an existing questionnaire were analyzed. This questionnaire was filled out by 1,685 general physicians (response rate $=47 \%$ ). The answers of these open-ended questions were coded inductively. Second, 21 semi-structured interviews (about experienced barriers and facilitators) were performed with physician SUD-
\end{abstract}

patients, significant others, and PHP employees. Themes identified in the first sub-study were used to deductively code the interview transcripts. Results were reported in accordance with the Consolidated Criteria for Reporting Qualitative Research guidelines. Results: Barriers were found at the level of the individual physician (negative feelings and lack of disease awareness), whereas facilitators were found at the level of social relationships (confrontation with SUD and social support) and health services (supportive approach, good accessibility, and positive image of services). The interviews emphasized the importance of nonjudgmental confrontation by social relationships in the process of seeking help for SUD. Conclusion: Physicians with SUD face barriers when seeking help for SUD mostly at the level of the individual physician. Health services and people around physicians with SUD could facilitate the help-seeking process by offering confidential and nonpunitive support. Future studies should explore whether the barriers and facilitators identified in this study also hold for other mental health issues.

(c) 2021 The Author(s)

Published by S. Karger AG, Basel
(C) 2021 The Author(s)

Published by S. Karger AG, Basel

This is an Open Access article licensed under the Creative Commons Attribution-NonCommercial-4.0 International License (CC BY-NC) (http://www.karger.com/Services/OpenAccessLicense), applicable to the online version of the article only. Usage and distribution for commercial purposes requires written permission. 


\section{Introduction}

The prevalence of unhealthy alcohol use among physicians is, according to data from the USA, slightly higher (15\%) than population prevalence rates $(13 \%)[1,2]$. In Europe, hazardous alcohol and drug use among physicians were estimated at 18-23 and 3\%, respectively [3-9]. Data on the prevalence of substance use disorders (SUDs) among physicians are limited. However, SUDs often cause tremendous personal harm and might also impair physicians' general functioning [10]. Consequently, SUD among physicians might affect quality of healthcare and patient safety, with studies suggesting mistakes in diagnosis and medical procedures, or problematic communication with patients as potential consequences in clinical practice $[11,12]$.

In the 1970s, the American Medical Association initiated Physician Health Programs (PHPs) aimed at promoting early identification, evaluation, and confidential and adequate treatment for physicians with SUD [13]. Over the past decades, PHPs have been set up in various Western countries across the globe (e.g., USA, Canada, Australia, New Zealand, United Kingdom, Spain, Norway, Switzerland, and Ireland) $[14,15]$. Our recent metaanalysis of PHP success rates showed an average abstinence rate of $72 \%$ and successful work resumption rate of $77 \%$ at follow-up [16]. In contrast, studies showed that among SUD patients in general relapse rates are around $50 \%$ [17-19].

Despite increasing availability of PHPs and their encouraging results, physicians hardly use health services when confronted with mental health problems, including SUD [20]. Before physicians access regular mental health care, they may face barriers at the individual level, like embarrassment and fear of possible consequences, but also on the level of health-care services, like worries about the quality of care and confidentiality $[21,22]$. Moreover, a questionnaire study among Finnish physicians $(N=$ 3,116 ) found that almost two-thirds self-medicated their mental disorder [23]. Furthermore, a qualitative study among Australian general practitioners showed that informal consultation at a colleague, without proper history-taking or physical examination, is also commonly practiced [22]. Together, these factors impede access to regular (mental) healthcare by physicians with mental health problems.

In the case of SUD, denial or minimization of substance use problems is an additional barrier for seeking help [24]. A systematic review of 8 qualitative and 23 quantitative studies among health-care professionals with SUD identified embarrassment and fear of possible consequences as major barriers and specific events, like drunk driving or a positive drug screen, and supportive relationships as major facilitators for seeking help [25]. However, few European studies were included (more than two-thirds were from the USA), less than half of the included studies described a sample of physicians, and all included studies solely covered the viewpoint of healthcare professionals with SUD, but not of significant others [25].

In the current study, we applied a qualitative methodology in order to identify barriers and facilitators when seeking help for SUD among Dutch physicians. To ensure a multiple perspective approach, we focused upon both anticipated and experienced barriers and facilitators and included 4 different participant groups. More specifically, we (1) explored anticipated barriers and facilitators to seek help for SUD among physicians in general using 2 open-ended questions of an existing questionnaire, and (2) investigated experienced barriers and facilitators when actually seeking help for SUD among physicianpatients, significant others, and PHP employees by means of semi-structured interviews.

\section{Materials and Methods}

\section{Data Collection}

Two qualitative methods were adopted to gather information about barriers and facilitators when seeking help for SUD among physicians: first, 2 open-ended questions about anticipated barriers and facilitators among general physicians were used from an existing online cross-sectional questionnaire (study 1) [26]. Second, semi-structured interviews with physician SUD-patients, significant others, and PHP employees were performed (study 2). The open-ended questions (study 1) were used to explore anticipated barriers and facilitators among physicians in general. The interviews (study 2) were used to more in-depth investigate distinct barriers and facilitators among physician-patients, significant others, and PHP employees based on their personal experiences with seeking help for SUD among physicians. The Consolidated Criteria for Reporting Qualitative Research guidelines were used to report the findings of this study, shown in online supplementary Table 1 (see www.karger.com/doi/10.1159/000517043 for all online suppl. material) [27].

\section{Study 1: Open-Ended Questions from Existing Questionnaire}

For a previous study [26], we used closed questions of an online cross-sectional questionnaire about "Addiction in physicians." This questionnaire was administered to the physician panel of the Royal Dutch Medical Association (RDMA) in September 2016. All panelists received an email with the invitation to participate and a link to the online questionnaire. They were informed about the nature of the questionnaire beforehand and they could decide to participate or not. Panelists had 3 weeks to complete the question- 
naire. They received 2 reminders to respond to the questionnaire. Encrypted data were collected via the web-based questionnaire platform. The questionnaire data were synthesized with encrypted demographic information of the panelists.

For this study, 2 open-ended questions about anticipated barriers and facilitators of this questionnaire were used. Prior to these open questions, respondents were instructed to hypothesize that they suffered from SUD themselves and that they intended to contact the Dutch PHP. Subsequently, they were asked: "What barriers would you experience (when intending to contact the Dutch PHP for your own SUD)?" and "What would help you to overcome these barriers?" The internal ethical review board of the RDMA reviewed and approved the questionnaire [26].

\section{Study 2: Semi-Structured Interviews}

Semi-structured interviews concerning "Help seeking for SUD by physicians" were prepared by means of a topic list, which was pilot-tested among 2 students (medicine and social sciences). The topic list was based on the themes identified by the open questions and was used as a guideline during the interviews, shown in Box S1, Box S2, and Box S3. A female researcher (P.M.G.) with a background in health sciences and trained in conducting interviews performed the semi-structured interviews between February and November 2019. Interviewees knew the background of the interviewer and that the interview was about the help-seeking process of physicians with SUD. Interviewees choose to conduct the interview by telephone or face-to-face (at their home or work, at the Department of Psychiatry of Radboudumc, or at the Dutch PHP). All interviews were audio recorded and transcribed verbatim. Field notes were made during the interview, and after each interview, the degree of saturation was discussed by the interviewer and her supervising senior researcher (A.F.A.S.). All interviewees gave their written informed consent prior to the start of each interview and were given the possibility to reflect and comment on the accuracy and validity of the obtained information afterward ("member checking").

Physician-patients and significant others were approached via employees of the national Dutch PHP, via clinicians and therapists affiliated to our regional addiction research consortium (Nijmegen Institute for Scientist-Practitioners in Addiction: NISPA), and via the Dutch self-help group Doctors Anonymous. By use of a recruitment text, we asked (former) physician-patients and significant others whether they wanted to participate in an interview about the help-seeking process. The researcher approached the PHP employees via e-mail. There was no pre-existing relationship between the researcher and physician-patients/significant others. One significant other withdrew her informed consent for an unspecified reason. The local Medical Ethical Committee of the Radboudumc reviewed and approved the interview study (registration number: 2019-5160).

\section{Sociodemographic Characteristics}

Gender and age were collected for all participants. Information on medical specialty, years in practice, and working situation was also available for physicians. Medical specialty was divided into 4 categories in line with literature [28]: general practice, (psycho) social medicine, contemplative somatic medicine, and surgical and supportive medicine, as we did previously [26].

\section{Data Analysis}

Descriptive statistics of participant characteristics were analyzed using the Statistical Package for Social Sciences software (SPSS version 22.0, IBM Corporation, Armonk, NY, USA). The text from the open-ended questions in the questionnaire and interview transcripts was coded using Atlas.ti software (version 8.3, Scientific Software Development GmbH). The first author (P.M.G.) coded the questionnaire answers inductively; the second author (E.P.) checked the analysis and discussed ambiguities with the first author (P.M.G.) until consensus was reached. The themes identified by the questionnaire analysis were used to code the interview transcripts deductively. The first and second author (P.M.G. and E.P.) independently coded 8 randomly chosen interviews, thereafter discrepancies were discussed until consensus was reached. The first author (P.M.G.) coded the remaining transcripts. Next, the first author (P.M.G.) clustered codes into themes, before the first, second, and last author (P.M.G., E.P., and A.F.A.S.) discussed the identified themes and adapted them if required.

\section{Results}

\section{Characteristics of Participants}

Study 1

The sample of panelists who responded to the questionnaire $(N=1,685$; response rate: $47 \%)$ consisted of general practitioners (34\%), (psycho) social physicians (28\%), contemplative somatic specialists (22\%), and surgical and supportive specialists (15\%) (shown in Table 1).

\section{Study 2}

Twenty-one interviews were conducted with ten physician-patients (substances of abuse: 60\% alcohol, 20\% opioids, $10 \%$ ketamine, and 10\% nicotine), 4 significant others (therapist, occupational physician, manager, and colleague), and 7 PHP employees (team members, advisors, and manager). Interviews took on average $55 \mathrm{~min}$ (range: 23-102 $\mathrm{min}$ ).

\section{Barriers and Facilitators}

Anticipated barriers and facilitators mentioned by general physicians (questionnaire respondents) could be classified at the level of the individual physician (5 themes), the level of social relationships (5 themes), and the level of health services ( 5 themes) (shown in Table 2). The majority of the general physicians anticipated one or more barriers $(N=1,153 ; 68 \%)$ and one or more facilitators $(N=1,094 ; 65 \%)$ for seeking help for SUD. The remaining respondents did not anticipate any barriers or facilitators to help seeking $(N=79 ; 5 \%$ and $N=10 ; 1 \%$, respectively), did not know what barriers or facilitators to anticipate $(N=123 ; 7 \%$ and $N=161 ; 10 \%$, respectively), or did not answer the question $(N=330 ; 20 \%$ and $N=$ 
Table 1. Sociodemographic characteristics of participants

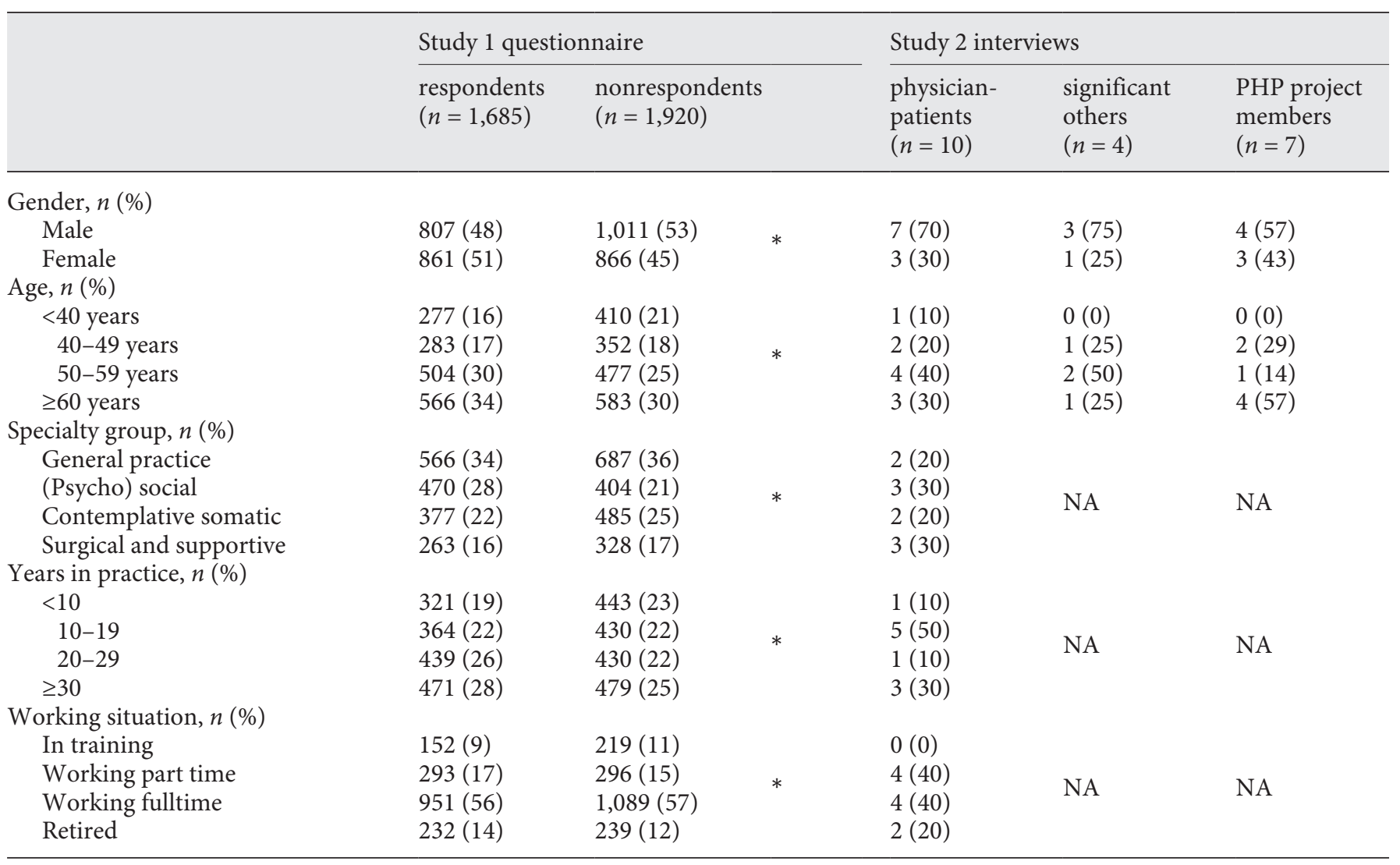

NA, not applicable; PHP, Physician Health Program. ${ }^{*} p<0.05$.

$420 ; 25 \%$, respectively). Results are presented as descriptions of the identified themes per level, supplemented with in-depth information from the interviews.

\section{Level 1: Individual Physician}

General physicians (questionnaire respondents) anticipated 4 out of 5 barriers for seeking help for SUD at the level of the individual physician (shown in Table 2). These barriers were associated with disease awareness, taking action, and consequences of disclosure. Respondents anticipated negative feelings like embarrassment, fear and anxiety, insecurity, and feelings of guilt, failure, powerlessness, or weakness. Questionnaire respondents mentioned these feelings often in combination with denial of signs and symptoms or not being aware of SUD. In addition, respondents expressed fear with regard to professional consequences like suspension or dismissal, report to the health inspectorate, and private problems. Over time, barriers can turn into facilitators. For example, awareness of possible consequences may ultimately result in seeking help for SUD. However, at the level of the individual patient barriers were much more often anticipated than facilitators.

Physician-patients, significant others, and PHP employees (interviewees) mentioned similar themes as compared to general physicians. All physician-patients mentioned lack of disease awareness, in combination with negative feelings according to two-thirds of the physician-patients, as main barrier(s) for seeking help.

Physician-patient \#6: "Substance use is associated with embarrassment. It would be hard going to my own GP, with whom I also work professionally... It would be less hard to me if I was depressed or had back pain."

PHP employees believed that professional consequences of disclosure prevent physician-patients from seeking help.

PHP employee \#3: "Many physicians don't want to put their work down or get it [their SUD] disclosed or go on sick leave ... Their fear includes losing everything, [their] work and partner..."
Geuijen/Pars/Kuppens/Schene/de Haan/ de Jong/Atsma/Schellekens 


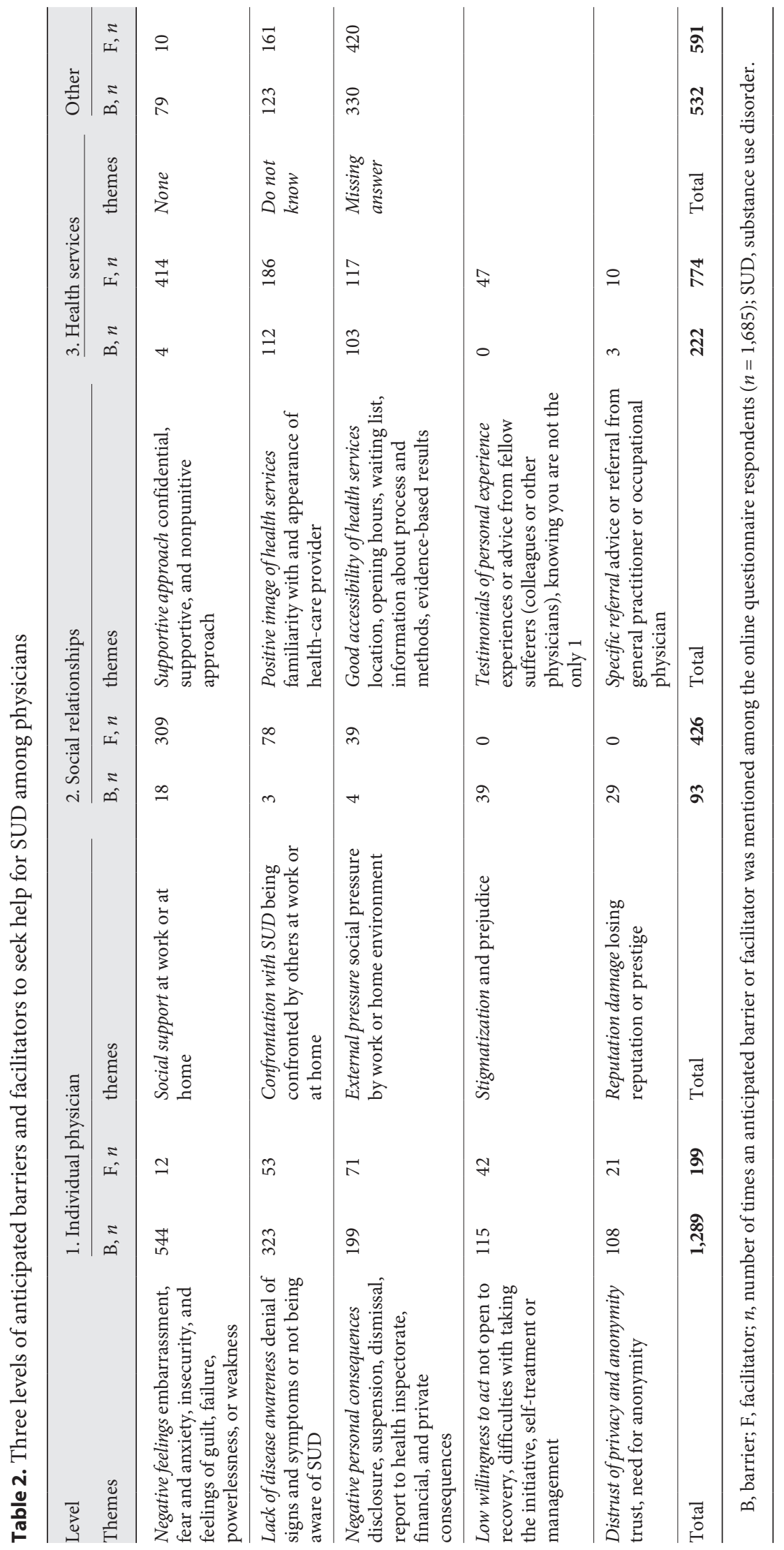


Additionally, the experiences of most physicianpatients, significant others, and PHP employees showed that it takes time to seek help because physicians with SUD have to overcome their procrastination and doubt regarding privacy and anonymity. Some PHP employees mentioned that physician-patients have difficulties with estimating the seriousness of the situation.

PHP employee \#3: "They think: I will be able to stop myself. That is most of the time true, they can stop themselves, but after a while they will get stuck."

According to physician-patients and significant others, physicians should receive training about the risks of SUD, self-care, and self-treatment during the medical curriculum, at conferences, and by journal articles.

Physician-patient \#7: "Attention should be payed to SUD in the medical curriculum ... whatever specialty you are going to do, you will encounter patients with SUD and also colleagues."

\section{Level 2: Social Relationships}

General physicians mentioned social relationships almost 5 times more often as a facilitator than as a barrier for seeking help. They anticipated the social network to facilitate help seeking by providing support and by confronting the physician-patient with the SUD. Support or confrontation from the working environment (colleague, supervisor, or employer) was more often anticipated as facilitator than support or confrontation from the physician-patient's home environment (partner, family member, or friend).

General physician \#248: "[I would need] a loved one or colleague who acknowledges my problem and who treats me with respect instead of judging me."

The interviews showed that discovery of the SUD by others (at work or at home) or by a specific event (positive urine screen at work, drunk driving) led to confrontation of the physician-patient with his or her problem. It was frustrating to some of the physician-patients that people in their environment had a presumption for some time, but they did not discuss it with the physician in question.

Physician-patient \#1: "Why did everyone shut up for three months?" To me, that is still a mystery... There was no one who confronted me. Apparently, people do not dare to do that..."

After nonjudgmental confrontation and/or disclosure, social support is highly valued by physician-patients. Social relationships could help the physician-patient by a combination of attentive listening, offering comfort, and practical and/or informative help. One of the significant others experienced the consequences of not offering social support.

Significant other \#4: "The physician in question who drank himself to death in my hospital, one year earlier he had already indicated that he could not take it anymore... his colleagues did not support him... he plodded through, with this [his death] as a result... After that [there were] feelings of guilt in that department...."

Significant others and PHP employees mentioned the importance of a preconceived plan for offering help.

Significant other \#4: "I think it is important that there is already a good plan ... [then you can] discuss this [the presumption of SUD] with more obligation than just saying "I worry about you."

Several physician-patients mentioned the themes stigmatization (prejudice) and reputation damage as barriers to seek help for their SUD. Some physician-patients were afraid that the way social relationships see and value them would be impaired by disclosure of their SUD. Half of the interviewees stated that external pressure also facilitates help-seeking, that is, social relationships (family members, employer, or health inspectorate) expect or obligate the physician-patient to be treated for his or her SUD.

\section{Level 3: Health Services}

Finally, several general physicians mentioned anticipated barriers to seek help for SUD at the level of health services (health-care providers or PHP), like no positive image of health services, and poor accessibility of health services. A nonpositive image included nonfamiliarity with and bad appearance of health services, whereas poor accessibility meant that the health service is difficult to approach. General physicians frequently anticipated facilitators at the level of the health services, such as a supportive approach, positive image and good accessibility of health services, and positive testimonials of personal experience.

All interviewees (physician-patients, significant others, and PHP employees) preferred that health services endorse a confidential, supportive, and nonpunitive approach with a focus on recovery. It was disappointing to some physicians that there were long waiting lists (6-8 months) at health-care providers, which delayed the process of treatment, recovery, and return to work.

Physician-patient \#1: "It is a shame that there are waiting lists of six to eight months everywhere ... I ended up sitting at home for almost a year... I don't think it was good for me to stay at home for so long."

According to the majority of physician-patients and PHP employees, the counseling service of the PHP was
Geuijen/Pars/Kuppens/Schene/de Haan/ de Jong/Atsma/Schellekens 
easily accessible because there was no waiting list, no direct allocation to treatment (and thereby no registration at the health insurer), while anonymous guidance is optional. To most physician-patients, it was important that health services share testimonials of recovered physicianpatients. By sharing these experiences, physician-patients feel and/or realize that they are not the only one.

Physician-patient \#6: "Use an ambassador as a role model, if he/she dares it ... preferably someone with stature: "If it happens to him/her, then I am not so special."

\section{Discussion}

This study aimed to identify barriers and facilitators with regard to seeking help for SUD among physicians from a multiple perspective approach. Our questionnaire and interview data showed that physicians with SUD face barriers for seeking help mainly at the level of the individual physician, like embarrassment, anxiety, and denial. In contrast, a supportive approach by health services and social relationships could facilitate help seeking. Furthermore, in the interviews, participants emphasized the importance of nonjudgmental confrontation by social relationships and sharing personal experiences of overcoming SUD in order to increase help seeking for SUD among physicians.

Behavioral change is a key element in overcoming SUD [29]. According to the transtheoretical model, behavioral change comes with 5 stages: precontemplation, contemplation, preparation, action, and maintenance [29]. In line with existing SUD literature, several barriers for seeking help at the level of the individual physician as identified in our study suggest that being in the precontemplation phase indeed hinders help seeking behavior $[24,29]$. In more detail, participants reported lack of acknowledgment of the SUD and low willingness to act as barriers for seeking help, which could both be seen as indicators of precontemplation.

Other identified barriers and facilitators were linked to disclosure. More specifically, others finding out about a physician's SUD and nonjudgmental confrontation with SUD were mentioned as potential facilitators for seeking help, by providing both social support and external pressure from social relationships. As result of disclosure, physicians with SUD might on the one hand experience negative feelings, stigmatization, and worries about their reputation, privacy, anonymity, and negative personal consequences at home and at work. On the other hand, nonjudgmental confrontation may increase awareness

Physicians Seeking Help for Substance Use Disorder and acknowledgment of the SUD, thus facilitating contemplation and potentially subsequent preparation for behavioral change [29]. Such an approach is very much in line with the principles of motivational interviewing (nonjudgmental and nonthreatening approach). Since a large body of evidence supports effectiveness of motivational interviewing for facilitating behavioral change and seeking help for SUD [30], education on motivational interviewing as part of the medical curriculum could impact on both patient care and interprofessional responsibility.

Though precontemplation, lack of SUD awareness and disclosure are also encountered by nonphysician SUD patients [21], an Australian qualitative study found that specifically physician-patients worry about formal report to the health inspectorate and loss of professional reputation or career development opportunities [31]. As a result, it could be speculated that in physician SUDpatients the precontemplation phase may last longer compared to nonphysician SUD-patients. Furthermore, their access to some substances of abuse (e.g., benzodiazepines and opioids), their usually comfortable financial situation, and intellectual abilities might further facilitate denial and the ability to disguise their SUD for a long time [25].

By combining hypothetical views from a large group of physicians with experiences of a small group of physicians and other relevant stakeholders, including significant others, with experience with seeking help for SUD, we comprehensively explored (potential) facilitators and barriers for seeking help in physicians with SUDs. Interestingly, these anticipated and experienced barriers and facilitators largely overlapped. The current findings also suggest that barriers for seeking help may in some turn into facilitators for seeking help. For instance, some respondents mentioned negative feelings as a barrier, whereas others anticipated negative feelings as a facilitator for seeking help for their SUD. Though this finding might suggest that barriers and facilitators are very personal, it might also be that perceived barriers might become facilitators, depending on the stage of change and level of support from the environment. Indeed, a systematic review argued that physicians' health-care access will benefit from cultural acceptance around physicians who seek help for mental health problems [21].

The majority of the facilitators identified in the current study was associated with (open) communication. More specifically, participants mentioned nonjudgmental confrontation with SUD, social support, and external pressure as means to facilitate disclosure of SUD and guiding 
someone toward SUD treatment. We previously observed that although most physicians (97\%) intend to act upon a substance use presumption in a colleague, only twothirds (65\%) actually took action in such case [26]. Therefore, physicians should be trained to recognize signs and symptoms of SUD and learn how to take appropriate action in case of a substance use presumption in a colleague [26]. Since confrontation from the physician's working environment was more often mentioned as facilitator than confrontation from the physician's home environment, workplace awareness campaigns might facilitate nonjudgmental identification of physicians struggling with SUD. In the Netherlands, the RDMA recently launched such an awareness campaign, which offers policy, education, and ambassadorship tools for employers [32].

Another important facilitator identified in the current study is sharing (positive) personal experiences by ambassadors, which was mentioned as a way to increase visibility of and familiarity with available SUD health services and to stimulate help-seeking. Thereby, clear information of the health-care provider or PHP about treatment facilities and treatment results could further lower the threshold for accessing health care and stimulate motivation for treatment. Clear information on available treatment options in combination with sharing testimonials of personal experiences by physicians who overcame their SUD will help physician-patients to become confident about the quality of care provided and the possibility to successfully overcome their SUD. Therefore, it is recommended that SUD health services commit former physician-patients as role models to their services. By sharing their testimonials, these role models might contribute to reducing barriers for help seeking.

Once a physician with an SUD takes the step for seeking help, it is important that employers or health-care providers respond adequately by offering referral to confidential treatment where needed and ensuring proper follow-up and work resumption after treatment [10]. When physician-patients access regular health care, studies suggest that the treating health-care professional should avoid assumptions about a physician-patient's knowledge or insight in the SUD [33]. It is recommended to make reference to his or her medical status, but to also account for someone's unique hopes, fears, and expectations [33]. Medical associations and employers should raise physicians' awareness about the risks of self- or informal treatment and emphasize the importance of professional support, which can further facilitate the recovery process [33]. Ideally, this professional support follows a confidential and supportive approach. Although the US PHPs also value confidentiality, especially European PHPs (e.g., in Norway, Spain, the United Kingdom, and the Netherlands) encourage voluntary help-seeking, which is associated with high rates of self-referrals (45$75 \%)[15,34]$.

Current literature about seeking help by health-care professionals mainly focused on general health problems among physicians [21] and seeking help for SUD in general health-care professionals [25]. We were specifically interested in help seeking for SUD among physicians. Furthermore, the existing literature mainly focused on barriers for seeking help from the single perspective of health-care professionals themselves. Our study used a multiple perspective (hypothetical and experiences) and methods approach for the identification of both barriers as well as facilitators for help seeking for SUD, with relatively large sample sizes in the questionnaire study. However, this study should also be interpreted in light of several limitations. Although the response rate of the questionnaire was acceptable, young physicians, females, and the surgical and supportive specialty group were underrepresented in our study when compared to the whole physician community in the Netherlands [35]. An American questionnaire study among a nationally representative sample of 5,829 physicians showed that younger and male physicians were more reluctant to seek help for mental health issues than older and female physicians [36]. The anticipated barriers identified in the current study may be specifically relevant for young and male physicians with SUD. However, future studies may explore differences in barriers and facilitators for seeking help for SUD among physicians of different age, gender, and specialty.

Another limitation is that sampling bias cannot be ruled out in the current study. Participants who chose to respond to the questionnaire invitation or took part in the interviews might have had personal reasons to do so, or might have had specific ideas concerning SUD among physicians compared to those who declined to participate. Furthermore, for the interviews, we mainly recruited participants who contacted the Dutch PHP, and to a lesser extent through addiction care facilities. Those physicians with SUD who did not seek any form of help were therefore not reached. As often the case, patients who do not engage in the health-care system are difficult to reach for both health-care and scientific research. Especially concerning SUD, experienced shame and stigma might further hinder openness about SUD among physicians. Yet, by including interviewees with a variety of demo- 
graphic characteristics, medical specialties, and working experience, we attempted to cover multiple perspectives, increasing the generalizability of this study. Since we only focused on perceived barriers and facilitators to help seeking for SUD, future research should investigate whether the barriers and facilitators found in this study, also hold for other mental health or physical problems among physicians, as SUD is often considered a highly stigmatized disorder.

To conclude, physicians with SUD perceive several barriers for seeking help for SUD mostly at the level of the individual physician, like negative feelings and denial of SUD. Health services and people around physicians with SUD could facilitate the help-seeking process by offering a supportive approach (confidential and nonpunitive), positive image and good accessibility of health services, and by nonjudgmental confrontation of the physicianpatient with his or her problem. Ambassadors sharing personal (positive) testimonials can further facilitate help seeking for SUD by physicians. In the end, effective identification and management of SUD among physicians not only improves the quality of life of the individual physician but also contributes to the quality of patient care. Future studies should explore whether the perceived barriers and facilitators for seeking help for SUD also hold for other mental health issues, like mood disorders, anxiety disorders, and burnout.

\section{Statement of Ethics}

All interviewees gave their written informed consent prior to the start of each interview. One significant other withdrew her informed consent for an unspecified reason. The local Medical Ethical Committee of the Radboudumc reviewed and approved the interview study (registration number: 2019-5160). The authors assert that all procedures contributing to this work comply with the ethical standards of the relevant national and institutional committees for human studies and with the World Medical Association Declaration of Helsinki.

\section{Conflict of Interest Statement}

Arnt F.A. Schellekens is an Editorial Board Member of the journal. Other than this the authors declare that they have no known competing financial interests or personal relationships that could have appeared to influence the work reported in this article.

\section{Funding Sources}

This work was supported by a grant of the Dutch Ministry of Health, Welfare, and Sport (grant number 328622) to the RDMA. The funders had no role in study design, collection, analysis, or interpretation of the data, writing the manuscript, or the decision to submit the article for publication.

\section{References}

1 SAMSHA. Results from the 2010 National Survey on drug use and health: vol. I. Summary of national findings. Rockville, MD: SAMSHA (Substance Abuse and Mental Health Services Administration); 2011.

2 Oreskovich MR, Shanafelt T, Dyrbye LN, Tan L, Sotile W, Satele D, et al. The prevalence of substance use disorders in American physicians. Am J Addict. 2015 Jan;24(1):30-8.

3 Rosta J, Aasland OG. Female surgeons' alcohol use: a study of a national sample of norwegian doctors. Alcohol Alcohol. 2005 SepOct;40(5):436-40.

4 Rosta J. Hazardous alcohol use among hospital doctors in Germany. Alcohol Alcohol. 2008 Mar-Apr;43(2):198-203.

5 Unrath M, Zeeb H, Letzel S, Claus M, Escobar Pinzón LC. Identification of possible risk factors for alcohol use disorders among general practitioners in Rhineland-Palatinate, Germany. Swiss Med Wkly. 2012;142:w13664.

6 Joos L, Glazemakers I, Dom G. Alcohol use and hazardous drinking among medical specialists. Eur Addict Res. 2013;19(2):89-97.
7 Sørensen JK, Pedersen AF, Bruun NH, Christensen B, Vedsted P. Alcohol and drug use among Danish physicians. A nationwide cross-sectional study in 2014. Dan Med J. 2015 Sep;62(9):A5132.

8 Sørensen JK, Pedersen AF, Vedsted P, Bruun $\mathrm{NH}$, Christensen B. Substance use disorders among Danish physicians: an explorative study of the professional socialization and management of colleagues with substance use disorders. J Addict Med. 2016 Jul-Aug;10(4): 248-54.

9 Pförringer D, Mayer R, Meisinger C, Freuer D, Eyer F. Health, risk behaviour and consumption of addictive substances among physicians: results of an online survey. J Occup Med Toxicol. 2018;13:27.

10 Wallace JE, Lemaire JB, Ghali WA. Physician wellness: a missing quality indicator. Lancet. 2009 Nov; 374(9702):1714-21.

11 Oreskovich MR, Kaups KL, Balch CM, Hanks JB, Satele D, Sloan J, et al. Prevalence of alcohol use disorders among American surgeons. Arch Surg. 2012 Feb;147(2):168-74.
12 Sendler DJ. Physicians working under the influence of alcohol: an analysis of past disciplinary proceedings and their outcomes. Forensic Sci Int. 2018 Apr;285:29-37.

13 DuPont RL, McLellan AT, Carr G, Gendel M, Skipper GE. How are addicted physicians treated? A national survey of physician health programs. J Subst Abuse Treat. 2009 Jul;37(1):1-7.

14 Brooks SK, Gerada C, Chalder T. Review of literature on the mental health of doctors: are specialist services needed? J Ment Health. 2011 Apr;20(2):146-56.

15 Braquehais MD, Tresidder A, DuPont RL. Service provision to physicians with mental health and addiction problems. Curr Opin Psychiatry. 2015 Jul;28(4):324-9.

16 Geuijen PM, van den Broek SJM, Dijkstra BAG, Kuppens JM, de Haan HA, de Jong CAJ, et al. Success rates of monitoring for healthcare professionals with a substance use disorder: a meta-analysis. J Clin Med. 2021 Jan; 10(2):264.

17 Miller WR, Walters ST, Bennett ME. How effective is alcoholism treatment in the United States? J Stud Alcohol. 2001 Mar;62(2):21120.
Physicians Seeking Help for Substance Use Disorder
Eur Addict Res 2022;28:23-32 DOI: $10.1159 / 000517043$ 
18 National institute on drug abuse. Bethesda, MD: 2012.

19 van Wamel A, Croes E, van Vugt M, van Rooijen S. Prevalentie, zorgaanbod, effectiviteit en trends in de verslavingszorg. In: Achtergrondstudie in opdracht van het college van Zorgverzekeringen. Utrecht: Trimbos Instituut; 2014.

20 Tyssen R. Health problems and the use of health services among physicians: a review article with particular emphasis on Norwegian studies. Ind Health. 2007 Oct;45(5):599-610.

21 Kay M, Mitchell G, Clavarino A, Doust J. Doctors as patients: a systematic review of doctors' health access and the barriers they experience. Br J Gen Pract. 2008 Jul;58(552): 501-8.

22 Kay M, Mitchell G, Clavarino A, Frank E. Developing a framework for understanding doctors' health access: a qualitative study of Australian GPs. Aust J Prim Health. 2012;18(2): 158-65.

23 Töyry S, Räsänen K, Kujala S, Aärimaa $M$, Juntunen J, Kalimo R, et al. Self-reported health, illness, and self-care among finnish physicians: a national survey. Arch Fam Med. 2000 Nov-Dec;9(10):1079-85.
24 Motta-Ochoa R, Bertrand K, Flores-Aranda J, Patenaude C, Brunelle N, Landry M, et al. A qualitative study of addiction help-seeking in people with different co-occurring mental disorders and substance use problems. Int $J$ Ment Health Addiction. 2017 Aug;15(4):88399.

25 Kunyk D, Inness M, Reisdorfer E, Morris H, Chambers T. Help seeking by health professionals for addiction: a mixed studies review. Int J Nurs Stud. 2016 Aug;60:200-15.

26 Geuijen P, de Rond M, Kuppens J, Atsma F, Schene A, de Haan H, et al. Physicians' norms and attitudes towards substance use in colleague physicians: a cross-sectional survey in the Netherlands. PLoS One. 2020;15(4): e0231084.

27 Tong A, Sainsbury P, Craig J. Consolidated criteria for reporting qualitative research (COREQ): a 32-item checklist for interviews and focus groups. Int J Qual Health Care. 2007 Dec;19(6):349-57.

28 van Boekel LC, Brouwers EP, van Weeghel J, Garretsen HF. Stigma among health professionals towards patients with substance use disorders and its consequences for healthcare delivery: systematic review. Drug Alcohol Depend. 2013 Jul;131(1-2):23-35.

29 Prochaska JO, DiClemente CC, Norcross JC. In search of how people change. Applications to addictive behaviors. Am Psychol. 1992 Sep; 47(9):1102-14.
30 Miller WR, Rollnick S. Talking oneself into change: motivational interviewing, stages of change, and therapeutic process. J Cogn Psychother. 2004;18(4):299-308.

31 Clough BA, March S, Leane S, Ireland MJ. What prevents doctors from seeking help for stress and burnout? A mixed-methods investigation among metropolitan and regionalbased australian doctors. J Clin Psychol. 2019 Mar;75(3):418-32.

32 Royal Dutch Medical Association. ABSartsen toolkit. 2020.

33 Fox FE, Taylor GJ, Harris MF, Rodham KJ, Sutton J, Scott J, et al. It's crucial they're treated as patients: ethical guidance and empirical evidence regarding treating doctor-patients. J Med Ethics. 2010 Jan;36(1):7-11.

34 Bruguera E, Heredia M, Llavayol E, Pujol T, Nieva G, Valero S, et al. Integral treatment programme for addicted physicians: results from the galatea care programme for sick physicians. Eur Addict Res. 2020;26(3):12230.

35 CIBG. Over het BIG-register: cijfers. 2021.

36 Dyrbye LN, West CP, Sinsky CA, Goeders LE, Satele DV, Shanafelt TD. Medical licensure questions and physician reluctance to seek care for mental health conditions. Mayo Clin Proc. 2017 Oct;92(10):1486-93. 